\title{
Repair of mitral subvalvular apparatus and a calcified left ventricle aneurysm
}

\author{
Kasra Shaikhrezai', Sanjeet Singh Avtaar Singh ${ }^{1}$, Karim Morcos $^{1}$, Steve Hunter ${ }^{2}$ \\ 'Department of Cardiothoracic Surgery, Golden Jubilee National Hospital, Glasgow G81 4DY, UK. \\ 2Department of Cardiothoracic Surgery, Northern General Hospital, Sheffield S5 7AU, UK.
}

Correspondence to: Dr. Sanjeet Singh Avtaar Singh, Department of Cardiothoracic Surgery, Golden Jubilee National Hospital, Glasgow G81 4DY, UK. E-mail: sanjeetsingh@nhs.net

How to cite this article: Shaikhrezai K, Singh SSA, Morcos K, Hunter S. Repair of mitral subvalvular apparatus and a calcified left ventricle aneurysm. Vessel P/us 2018;2:9. http://dx.doi.org/10.20517/2574-1209.2018.17

Received: 29 Mar 2018 First Decision: 28 Apr 2018 Revised: 4 May 2018 Accepted: 7 May 2018 Published: 10 May 2018

Science Editor: Cristiano Spadaccio, Mario F. L. Gaudino Copy Editor: Jun-Yao Li Production Editor: Huan-Liang Wu

\begin{abstract}
Left ventricle (LV) myocardial infarction may result in changes to the structure of the subvalvular apparatus. This may lead to a functional regurgitation if accompanied by annular dilatation preventing coaptation of leaflets. Scar tissue formation in the left ventricle may also lead to aneurysm of the left ventricle. This can then calcify, making repair of the leaflet technically challenging. We present a case of a mitral valve repair with concomitant repair of left ventricle aneurysm in a 75-year-old gentleman who suffered an ST-segment elevation myocardial infarction to the lateral wall 20 years ago. He presented with breathlessness on minimal activity, severe mitral regurgitation with a posteriorly oriented regurgitant jet and calcification of LV aneurysm on chest X-ray and computed tomography scan. Despite the challenging nature, it is possible to repair a mitral valve with concomitant calcified LV aneurysm formation. Long term outcomes are still unknown for this cohort of patients.
\end{abstract}

Keywords: Transmural infarct, left ventricular dilatation, ischaemic mitral valve repair, left ventricular aneurysm with calcification

\section{INTRODUCTION}

Left ventricle (LV) myocardial infarction (MI) may initiate a series of configurational remodelling of the ventricle which leads to changes in the $3 \mathrm{D}$ geometry of the subvalvular apparatus ${ }^{[1]}$. This chain of events accompanied by annular dilatation may cause functional mitral regurgitation (MR). Another wellrecognised post MI complication is the LV aneurysm which may result in calcification of the aneurysm that can be visualised on a chest radiograph ${ }^{[2]}$. Generally LV aneurysm is considered as a deleterious outcome

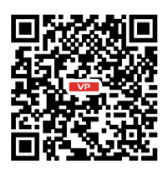



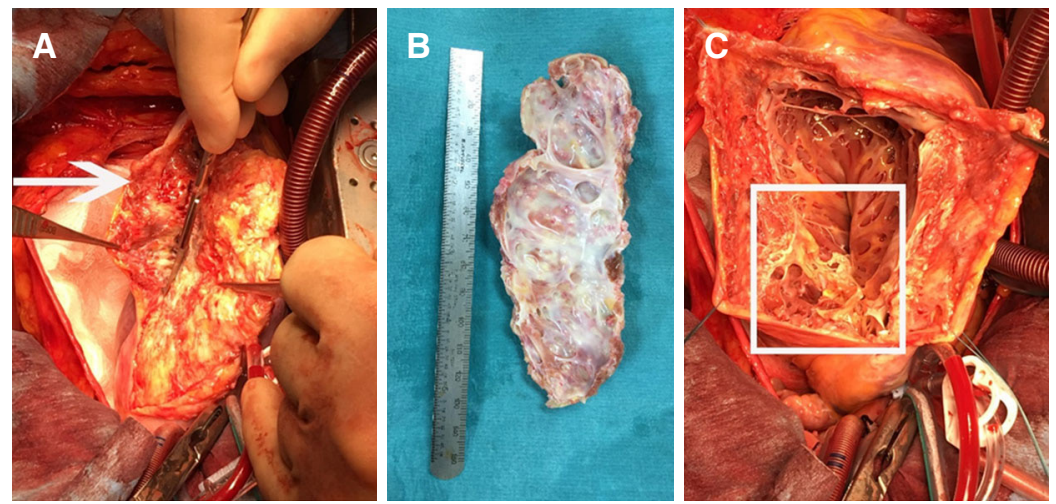

Figure 1. A: Shaving the calcified patch off the epicardium (arrow); B: the giant calcified patch; C: ventriculotomy with distorted and damaged mitral subvalvular apparatus (inside the square)

of MI with an increased risk of congestive heart failure, thromboembolism, unstable angina as well as ventricular tachycardia ${ }^{[3]}$. In symptomatic patients, aneurysmectomy is predominantly offered as an adjunct to surgical revascularisation however in this group the survival is more dependent on the revascularisation rather than the aneurysmectomy ${ }^{[3]}$. Combination of LV aneurysm calcification and severe MR remains a rare and challenging post-MI pathology while the failing heart on maximal medical therapy makes complex surgery the primary treatment choice.

\section{CASE REPORT}

A 75-year-old Caucasian male was referred with severe symptomatic MR 20 years following lateral STelevation MI. He presented with breathlessness on minimal activity, severe MR with a posteriorly oriented regurgitant jet and calcification of LV aneurysm on chest X-ray and computed tomography scan. His calculated EuroSCORE II mortality was $21 \%$.

Following institution of aorto-bicaval cardiopulmonary bypass (CPB), cooling to $32{ }^{\circ} \mathrm{C}$ and cardioplegic arrest, the heart was positioned to expose the lateral surface where the aneurysm was located. Using a skin knife (blade size 24), the lateral surface of the LV was incised longitudinally parallel to the course of posterior descending artery. An incision was made whereby the blade met the outer surface of calcification which was extended to endocardium and myocardium sparing the epicardium. The epicardium was shaved off the calcified patch [Figure $1 \mathrm{~A}$ ]. The patch measuring $15 \mathrm{~cm} \times 5 \mathrm{~cm}$ was entirely excised completing the ventriculotomy [Figure $1 \mathrm{~B}$ and $\mathrm{C}$ ]. The mitral subvalvular apparatus needed reconstruction due to excision of the calcified patch and intrinsic disease. The construction of subvalvular apparatus started with reimplantation of healthy parts of the posteromedial papillary muscle using pledgeted CV-4 polytetrafluoroethylene (PTFT) sutures (GORE-TEX, WL Gore \& Associates Inc, Flagstaff, AZ, USA) followed by attachment of neo-chordae to the edge of both mitral valve leaflets where detached native chordae were located. For papillary muscle reimplantation the fibrosed segment of papillary muscle was used to anchor a pledgeted box stitch to the stable part of papillary muscle already located on the LV. Subsequently the PTFT sutures were passed through the LV [Video 1] and tied on the outside of the heart using pledgets for more strength.

The neo-chordae to the edge of leaflets were left untied on the atrial side because prior to closure of ventriculotomy accurate length measurement of neo-chordae was impossible. Satisfactory reconstruction of subvalvular apparatus was confirmed by firm and stable reimplantation of papillary muscle and attachment of neo-chordae between papillary muscle and the leaflets for each detached native chordae. The ventriculotomy was closed by bovine pericardial patch which was sewn by running polypropylene suture around the edge of ventriculotomy [Figure $2 \mathrm{~A}$ ]. The epicardium flap from which the calcified patch was 

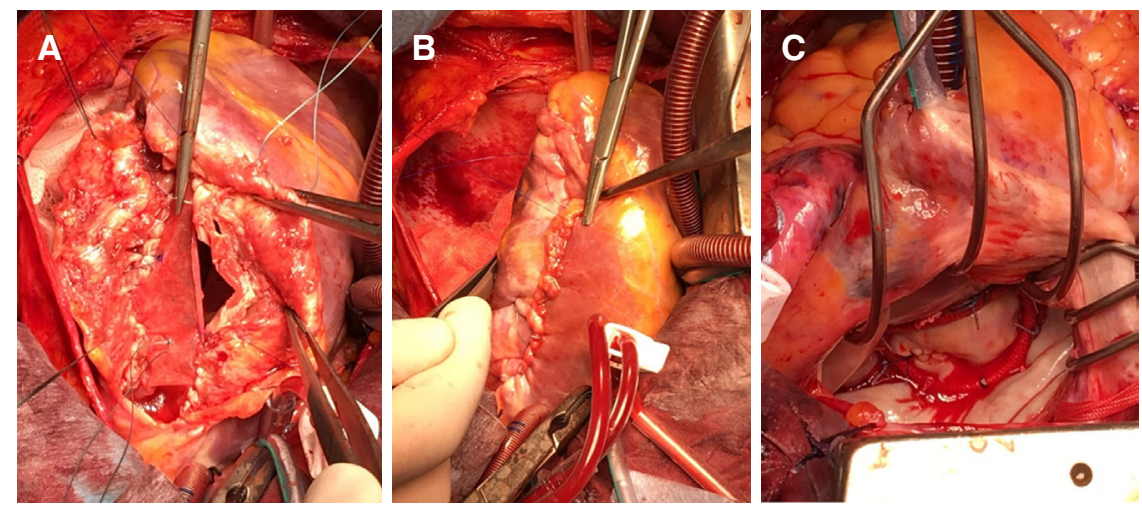

Figure 2. A: Closing the left ventricle by bovine pericardial patch; B: reinforcement of ventriculotomy closure by epicardium; C: mitral valve with ring annuloplasty is satisfactorily tested

shaved off was used to cover bovine pericardial patch for haemostasis [Figure 2B].

The atrial surface of mitral valve was approached via left atriotomy. The length of neo-chordae attached to the leaflets was accurately measured for an optimised coaptation before tying on the atrial side of the leaflets. A flexible 31-mm annuloplasty ring (St. Jude Medical Inc, St. Paul, MN, USA) was implanted after sizing the annulus from trigone to trigone and considering the area of anterior leaflet. The mitral valve was tested using a cardioplegia catheter into the LV through the valve [Figure $2 \mathrm{C}$ ]. This was confirmed following weaning from CPB. The patient was asymptomatic at 6 weeks and 3 months follow-up with an echocardiogram demonstrating mitral valve competence with improved LV function.

The overall cardiopulmonary bypass time was $158 \mathrm{~min}$ with an aortic cross clamp time of $133 \mathrm{~min}$.

\section{DISCUSSION}

LV aneurysm in the context of ischemic heart disease and subsequent calcification has been reported previously ${ }^{[4]}$. However, there is a paucity of literature regarding the surgical treatment of calcified LV aneurysm in a symptomatic patient with severe MR. LV aneurysm calcification can be attributed to chronic renal failure ${ }^{[5]}$ or other hypercalcemic conditions, but this was absent in our patient. He was also suffering from concomitant severe MR which was structurally distorted by the calcification of LV aneurysm. Although severe MR in a symptomatic patient would be an indication for surgery, we could not ignore the role of calcification, impairing valve function and its impact on the planned repair as without excision of the calcified patch reimplantation of papillary muscle and subvalvular apparatus repair was not feasible. Although concomitant mitral valve repair and excision of LV aneurysm calcification is possible with desirable short-term results, longer follow-up is required to evaluate outcomes.

\section{DECLARATIONS}

\section{Authors' contributions}

Performed the operation: Shaikhrezai K, Hunter S

Wrote the case report: Shaikhrezai K

Reviewed and made changes to the structure and format of the manuscript: Singh SSA

Reviewed the manuscript: Morcos K

Served as the primary supervisor of the manuscript: Hunter $\mathrm{S}$

Financial support and sponsorship

None. 


\section{Conflicts of interest}

The authors do not have any conflicts of interest to declare.

\section{Patient consent}

Patient consent (images/case history) was obtained during the consent process.

\section{Ethics approval}

The study was approved by the local Clinical Governance and Research and Development Unit.

\section{Copyright}

(c) The Author(s) 2018.

\section{REFERENCES}

1. Otsuji Y, Handschumacher MD, Schwammenthal E, Jiang L, Song JK, Guerrero JL, Vlahakes GJ, Levine RA. Insights from threedimensional echocardiography into the mechanism of functional mitral regurgitation: direct in-vivo demonstration of altered leaflet tethering geometry. Circulation 1997;96:1999-2008.

2. MacGregor JH, Chen JT, Chiles C, Kier R, Godwin JD, Ravin CE. The radiographic distinction between pericardial and myocardial calcifications. AJR Am J Roentgenol 1987;148:675-7.

3. Friedman BM, Dunn MI. Postinfarction ventricular aneurysms. Clin Cardiol 1995;18:505-11.

4. Lee BK, Atwood E. Images in clinical medicine. Calcified left ventricular aneurysm. N Engl J Med 2003;348:918.

5. Kempf AE, Momeni MG, Saremi F. Myocardial calcinosis in chronic renal failure. J Radiol Case Rep 2009;3:16-9. 